\title{
RF Transimpedance Amplifier for CATV Applications over PON
}

\author{
Guillermo Royo, Carlos Sánchez-Azqueta, Concepción Aldea and Santiago Celma \\ Group of Electronic Design (GDE) \\ Instituto de Investigación en Ingeniería de Aragón (I3A) \\ Universidad de Zaragoza, Mariano Esquillor s/n, 50018, Zaragoza, Spain. \\ Tel. +34-976762707, e-mail: royo@unizar.es
}

\begin{abstract}
In this work, we present a fully differential transimpedance amplifier (TIA) with controllable transimpedance for use in RF overlay downstream communication systems. The transimpedance amplifier has been designed in a standard $180-\mathrm{nm}$ CMOS technology and it is intended for $47 \mathrm{MHz}$ to $870 \mathrm{MHz}$ subcarrier multiplexed RF signals. It performs a $18 \mathrm{~dB} \Omega$ transimpedance gain control range for extended optical input range from $-6 \mathrm{dBm}$ up to $+2 \mathrm{dBm}$.
\end{abstract}

\section{Introduction}

The growing demand for information is driving continuous innovation in the design of communication systems. Optical links can transmit data at very high transmission rates, offering Internet, telephone lines and TV with just one network. The gigabit passive optical networks (GPON) provide a large bandwidth to fixed users with FTTx architectures (Figure 1). The GPON standard works with wavelengths of 1480-1500 nm for downstream and 1260-1360 nm for upstream. However, the upcoming $4 \mathrm{~K} / 8 \mathrm{~K}$ ultra high definition (UHD) contents will require a huge amount of data, significantly reducing the bandwidth reserved for Internet contents. Transmitting the UHD contents in a different optical wavelength allows to maintain high bandwidth and avoids interferences between the video and IP signal. This approach, known as RF overlay, adds a second downstream channel to provide video and TV services with RF signals, using the wavelengths reserved for community access TV (CATV) in GPON (1550-1560 nm), in a point-tomultipoint broadband communication system. With this technique, the transmission of UHD contents does not reduce the Internet bandwidth, as they are transmitted in different optical wavelengths, which do not interfere with each other. The different wavelengths are split using an optical triplexer once they reach the user's optical network terminal (ONT), where the RF contents are converted to the electrical domain. For RF overlay applications, the overall communication system, and specially the optical receiver must feature very low noise and high linearity to provide a signal with enough quality for the user. In addition, the different stages of the receiver must compensate the channel limitations. On the one hand, due to the attenuation of the optical fiber, the input power may vary a few $\mathrm{dBm}$ for different users. In order to compensate this variation and provide the same RF power to all users, an automatic gain control circuit (AGC) is needed. On the other hand, the losses of the coaxial cable that connects the ONT to the user's TV are 2-4 dB higher at high frequencies, which must be compensated with an equalizer (EQ), so that all the subcarriers reach the user's TV with the same RF power [1]. Fig. 2a shows the block diagram of a CATV receiver front-end. This work focuses on the design of the first block of the receiver, the transimpedance amplifier (TIA).

\section{Circuit Design}

The proposed TIA has been designed in a standard 180 -nm CMOS technology using a single $1.8-\mathrm{V}$ supply voltage. The topology consists of a shuntshunt feedback TIA that presents both low input impedance and low input noise. To fulfill the stringent linearity and noise requirements of CATV applications, a fully differential structure has been chosen to minimize the harmonic distortion and the intermodulation between adjacent channels. To maintain the stability of the system, an open loop gain control has been employed [2]. The implementation of the TIA consists of two cascaded differential pairs with a double control of both the transimpedance and the open loop gain, as shown in Fig. 2b. The transimpedance control is realized with a variable feedback resistor, while the open loop gain will be controlled with a variable load resistor in the first differential pair.

In order to achieve an optimum linearity, the variable feedback resistor has been implemented with a parallel combination of a resistor, $R_{F}$, and a digitally controlled PMOS transistor array. Using a digital control maximizes the overdrive voltage, so that the transistors work in the ohmic region, improving the linearity of the TIA compared to an analog control 
[3]. Similarly, with another resistor, $R_{L}$, in parallel with a MOS transistor array, a variable load resistor in the first differential pair to control the open-loop gain has been implemented.

\section{Results}

The transimpedance amplifier with automatic gain control has been designed in a standard 180-nm CMOS technology. The photodetector $(0.9 \mathrm{~A} / \mathrm{W}$ at $1550 \mathrm{~nm}$ ) and the biasing circuitry of a typical lowcost FTTH triplexer have been modeled as a current source in parallel with a $0.9 \mathrm{pF}$ capacitor in series with a $6 \mathrm{nH}$ inductance of the bonding wires. Fig. 3 shows the frequency response of the proposed TIA. The TIA achieves a transimpedance range from $48 \mathrm{~dB} \Omega$ to $66 \mathrm{~dB} \Omega$, with a $3-\mathrm{dB}$ bandwidth slightly higher than $1.5 \mathrm{GHz}$, featuring a frequency response flatness of $0.5 \mathrm{~dB}$ from $47 \mathrm{MHz}$ to $870 \mathrm{MHz}$. With a power consumption of only $27 \mathrm{~mW}$ from a $1.8-\mathrm{V}$ supply voltage, the equivalent input noie (EIN) is below $6 \mathrm{pA} / \sqrt{\mathrm{Hz}}$ in the frequency range of interest at the highest transimpedance. With this EIN level and a $0.9 \mathrm{~A} / \mathrm{W}$ responsivity photodiode, the minimum input signal power can be as low as $-6 \mathrm{dBm}$ to achieve a carrier-to-noise ratio high enough to recover the signal with a good quality.

\section{Conclusions}

A fully differential transimpedance amplifier with automatic gain control for use in CATV RF overlay applications is presented. Designed in a standard 180-nm CMOS technology with a supply voltage of $1.8 \mathrm{~V}$, the TIA presents an $18 \mathrm{~dB} \Omega$ transimpedance gain control. An adaptive control of the open-loop gain has been implemented, in order to achieve a flat frequency over the frequency range of interest and to maintain the stability of the system. The EIN level at the maximum transimpedance is lower than

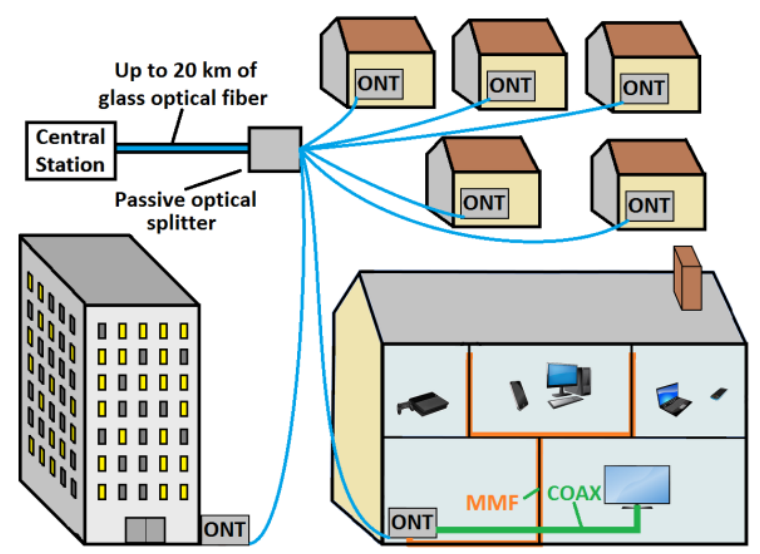

Fig. 1. Conceptual scheme of a passive optical network.
$6 \mathrm{pA} / \sqrt{\mathrm{Hz}}$, which allows the TIA achieve an input optical power range from $-6 \mathrm{dBm}$ to $+2 \mathrm{dBm}$, similar to that of the recently published GaAs PHEMT based receivers.

\section{Acknowledgements}

This work has been partially supported by MINECOFEDER (TEC2014-52840-R) and MINECO fellowship program to G. Royo (BES-2015-073748)

\section{References}

[1] BRILLANT, A. Digital and analog fiber optic communications for CATV and FTTX applications, Wiley-Interscience, 2008.

[2] SANZ, M., DEL POZO, J., CELMA, S., and SARMIENTO, A. Constant-bandwidth adaptive transimpedance amplifier, Electronics Letters, 2007 , 43(25) 1451-1452.

[3] ROYO, G., SÁNCHEZ-AZQUETA, C., GIMENO, C., ALDEA, C., and CELMA, S. CMOS transimpedance amplifier with controllable gain for RF Overlay, Proceedings of $12^{\text {th }}$ Conference on PhD Research in Microelectronics and Electronics, 2016.

(a)

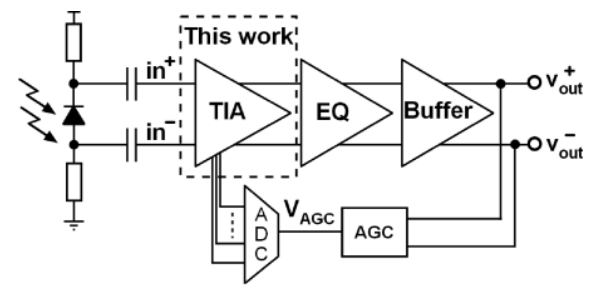

(b)

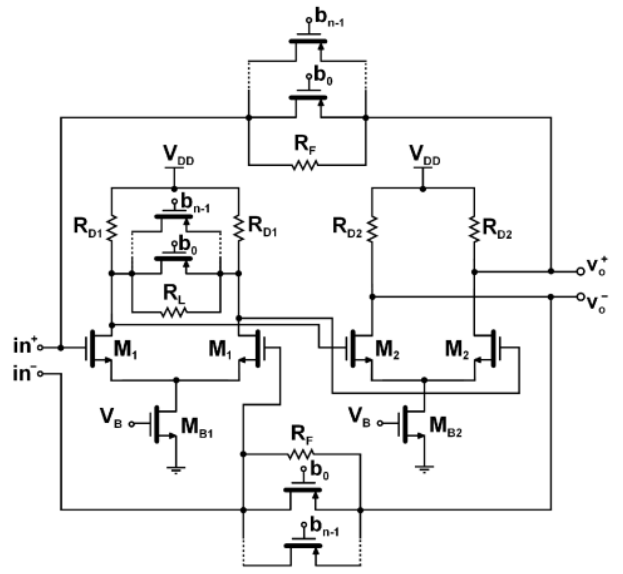

Fig. 2. a) Block diagram of a receiver front-end for RF overlay and b) schematic circuit of the proposed TIA.

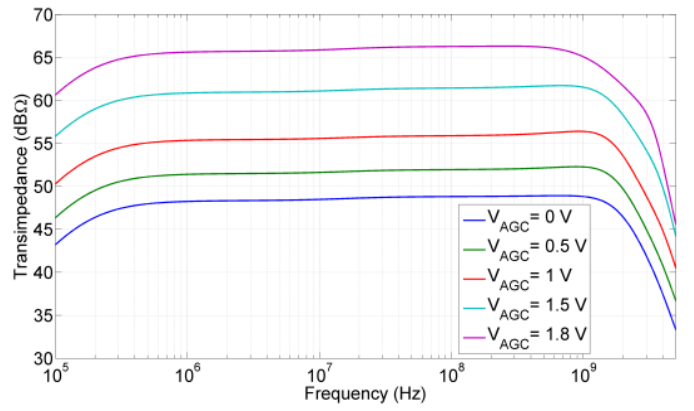

Fig. 3. Frequency response of the TIA.

Revista "Jornada de Jóvenes Investigadores del I3A", vol. 5 (Actas de la VI Jornada de Jóvenes Investigadores del I3A - 2 de junio de 2017). ISSN 2341-4790. 